\title{
Contamination of Mesenchymal Stem-Cells with Fibroblasts Accelerates Neurodegeneration in an Experimental Model of Parkinson's Disease
}

\author{
Marcia C. L. Pereira • Mariane Secco • Daniela E. Suzuki • Luciana Janjoppi • \\ Carolina O. Rodini • Layla B. Torres • Bruno H. S. Araújo • Esper A. Cavalheiro • \\ Mayana Zatz • Oswaldo Keith Okamoto
}

Published online: 19 April 2011

(C) The Author(s) 2011. This article is published with open access at Springerlink.com

\begin{abstract}
Pre-clinical studies have supported the use of mesenchymal stem cells (MSC) to treat highly prevalent neurodegenerative diseases such as Parkinson's disease (PD) but preliminary trials have reported controversial results. In a rat model of PD induced by MPTP neurotoxin, we first observed a significant bilateral preservation of dopaminergic neurons in the substantia nigra and prevention of motor deficits typically observed in PD such as hypokinesia, catalepsy, and bradykinesia, following intracerebral administration of human umbilical cord-derived MSC (UC-MSC) early after MPTP injury. However,
\end{abstract}

Electronic supplementary material The online version of this article (doi:10.1007/s12015-011-9256-4) contains supplementary material, which is available to authorized users.

M. C. L. Pereira • D. E. Suzuki · L. Janjoppi · C. O. Rodini • L. B. Torres · B. H. S. Araújo · E. A. Cavalheiro • O. K. Okamoto Disciplina de Neurologia Experimental,

Escola Paulista de Medicina, Universidade Federal de São Paulo, São Paulo, SP, Brazil

M. Secco $\cdot$ M. Zatz

Human Genome Research Center, Department of Genetics and Evolutive Biology, University of São Paulo,

São Paulo, SP, Brazil

L. B. Torres

Instituto Evandro Chagas - IEC- Centro Nacional de Primatas, Ananindeua, PA, Brazil

Present Address:

O. K. Okamoto $(\bowtie)$

Centro de Estudos do Genoma Humano, Departamento de

Genética e Biologia Evolutiva, Instituto de Biociências,

Universidade de São Paulo,

Rua do Matão 277, Cidade Universitária,

Caixa Postal 05508-090 São Paulo, SP, Brazil

e-mail: keith.okamoto@usp.br surprisingly, administration of fibroblasts, mesenchymal cells without stem cell properties, as a xenotransplantation control was highly detrimental, causing significant neurodegeneration and motor dysfunction independently of MPTP. This observation prompted us to further investigate the consequences of transplanting a MSC preparation contaminated with fibroblasts, a plausible circumstance in cell therapy since both cell types display similar immunophenotype and can be manipulated in vitro under the same conditions. Here we show for the first time, using the same experimental model and protocol, that transplantation of UC-MSC induced potent neuroprotection in the brain resulting in clinical benefit. However, co-transplantation of UC-MSC with fibroblasts reverted therapeutic efficacy and caused opposite damaging effects, significantly exacerbating neurodegeneration and motor deficits in MPTPexposed rats. Besides providing a rationale for testing UC-MSC transplantation in early phases of PD aiming at delaying disease progression, our pre-clinical study suggests that fibroblasts may be common cell contaminants affecting purity of MSC preparations and clinical outcome in stem cell therapy protocols, which might also explain discrepant clinical results.

Keywords Mesenchymal stem cells · Fibroblasts . Umbilical cord · Parkinson's disease · Cell therapy

\section{Introduction}

Stem cell-based neuroprotective and neurorestorative processes are among the latest therapeutic strategies currently under investigation for Parkinson's Disease (PD). Early clinical studies on allogeneic transplantation of fetal 
mesencephalic cells in the striatum have shown promising results, including increase in dopamine synthesis and attenuation of rigidity and bradykinesia in transplanted patients [1, 2]. However, further randomized, double blinded and placebo controlled clinical trials have not found significant motor improvements, and reported adverse effects such as dystonia and dyskinesia [3, 4]. More recently, scientific discussions regarding new strategies for cell therapy in PD have resumed in part due to recent advances in stem cell research and cumulative clinical experience [5-7], but divergent opinions prevail. Testing of alternative types of cells for transplantation, quality criteria for clinical grade cells, dose regimen and infusion routes, in addition to therapeutic and possible adverse effects are examples of fundamental questions that still need to be addressed at a pre-clinical stage to help designing future clinical trials for PD treatment.

Animal models of PD have been helpful in studying the neuropathological aspects of the disease and in testing novel therapeutic approaches, including transplantation of human adult [8-11] and embryonic stem cells (ESC) [12]. Pre-clinical studies with human neural stem cells and ESC have been performed in murine and non-human primate models with the primary aim of reestablishing the dopaminergic system, either by direct replacement of nigrostriatal neurons or by synthesis of dopamine by the exogenous cells [13-15]. Mouse iPS (induced pluripotent stem) cells have also been reported capable of generating neurons and glial cells both in vitro and in vivo, with functional recovery effects in a murine model of PD [16].

From these encouraging findings, ensuing practical issues emerge and should be considered from the clinical viewpoint. The use of pluripotent ESC and iPS cells is currently challenging due to ethical, technological, and safety reasons. In addition to the potential development of teratomas after transplantation of pluripotent cells, in the case of genetically reprogrammed cells, it remains unclear whether iPS cells derived from PD patients would generate dysfunctional neurons due to inherited genetic alterations associated with the disease [17]. This concern also applies to the use of endogenous stem and progenitor cells residing in the patient's own tissues. Therefore, obtaining genetically compatible human neural stem cells and/or neuroprogenitors for allogeneic transplantation is demanding. Other potential adverse effects of the transplantation of pluripotent cells and neuroprogenitors under consideration include improper neurotransmitter balance and development of aberrant neural connections that may lead to seizures, inhibition or hyperactivation of pre-existing neural circuitry, with impacts in cognition and behavior.

In addition to neuronal replacement, neuroprotective homeostatic adjustments within the cellular niche are also important to preserve cell function, tissue integrity, and physiological balance. Such indirect neuroprotective effects might be achieved through paracrine effects of the engrafted cells involving production of anti-inflammatory, angiogenic, and neurotrophic factors, favoring a repairing microenvironment within the central nervous system [18]. Indeed, post mortem analysis of PD patients subjected to allogeneic transplantation of fetal mesencephalic cells revealed that implanted cells eventually succumb to the degenerating environment in the patient's brain [19], indicating the therapeutic relevance of ameliorating the cell niche in a diseased brain.

Previous studies have shown that the human umbilical cord (UC) tissue is richer in mesenchymal stem cells (MSC) than its respective umbilical cord blood (UCB) [20]. In addition, the UCB- and UC-derived MSC express distinct repertoires of genes, the latter being enriched in transcripts of genes related to angiogenesis and neurogenesis [21]. Furthermore, MSC are poorly immunogenic lacking constitutive HLA-class II expression and have immunosuppressive activity [22]. Such molecular trait and immunomodulatory attributes of UC-MSC might be beneficial in cell therapy protocols for neurovascular and chronic neurodegenerative diseases, either in auto- or allotransplants.

Here, in a first experiment, we investigated the therapeutic potential of human UC-MSC in a rat model of PD induced by the neurotoxin 1-methyl, 4-phenyl, 1, 2, 3, 6tetrahydropyridine (MPTP). Distinct intracranial routes of administration were tested for cell engraftment. Preservation of dopaminergic neurons in the substantia nigra (SN) and improvements in motor function were considered as therapeutic end points, while anxiety and absence of visible tumor mass were specifically examined as potential adverse effects. Intracranial infusion of fibroblasts was performed as an additional xenotransplantation control of human cells without stem cell properties.

Transplantation of UC-MSC was well tolerated and a significant and specific therapeutic effect was achieved. To our surprise, while control animals infused with saline showed no effects, animals injected with fibroblasts presented exacerbated neurodegeneration and motor deficits. This observation prompted us to investigate if contamination of MSC preparations with fibroblasts, which express several markers in common with MSC, could have a detrimental as opposed to a beneficial therapeutic effect. In order to address this question, we performed additional transplant experiments using UC-MSC mixed with fibroblasts. Under such experimental conditions, treatment with human UC-MSC/fibroblasts did not result in therapeutic benefit and worsen the neurotoxic and behavioral effects of MPTP. Possible adverse effects due to poor purity of MSC preparations have not been properly addressed and they might provide an explanation for discrepant results in pre- 
clinical and clinical studies observed by different research groups.

\section{Material and Methods}

Isolation and Propagation of Human UC-Derived MSCs

The study was approved by the local institutional review boards. Ten human UC units were collected after obtaining informed consent from the donors. MSC were isolated and characterized in vitro as previously described [21]. Briefly, UCs were filled with $0.1 \%$ collagenase (Sigma-Aldrich, St. Louis) in phosphate-buffered saline (PBS) and incubated at $37^{\circ} \mathrm{C}$ for $20 \mathrm{~min}$. Each UC was washed with proliferation medium consisting of DMEM-LG, 10\% fetal bovine serum (FBS), $100 \mathrm{U} / \mathrm{mL}$ penicillin and $1 \%$ penicillin/streptomycin. Detached cells were harvested by gently massaging the UC. Cells were centrifuged at $300 \mathrm{~g}$ for $10 \mathrm{~min}$, resuspended in proliferation medium, and seeded in $25 \mathrm{~cm}^{2}$ flasks at a density of $5 \times 10^{7}$ cells $/ \mathrm{mL}$. After $24 \mathrm{~h}$ of incubation at $37^{\circ} \mathrm{C}$ in $5 \% \mathrm{CO}_{2}$, non-adherent cells were removed, and culture medium was replaced every 3 days. Adherent cells (third passage, at $80-90 \%$ confluence) were subjected to adipogenic, chondrogenic, and osteogenic differentiation in vitro according to established protocols [22], for multipotency testing.

Dermal fibroblasts were obtained from skin biopsies of healthy adult women after informed consent. Skin samples were dissected, sliced into $2-3 \mathrm{~mm}^{2}$ pieces with sterile scalpel and forceps, and transferred to cell culture dishes (Corning) in DMEM High Glucose medium, supplemented with $20 \%$ of FBS and $1 \%$ of Antibiotics (GIBCO, Invitrogen). Culture dishes were incubated at $37^{\circ} \mathrm{C}$ and $5 \% \mathrm{CO}_{2}$ and left undisturbed for $48 \mathrm{~h}$ to prevent tissue dislodging. The growth medium was changed every 3 days. Fibroblasts began to outgrowth from skin explants after 7 8 days of culture. When cells were about $70-80 \%$ confluent, they were dissociated from the dish surface using a trypsin solution (TryPLE Express; GIBCO, Invitrogen) and subcultured in DMEM High Glucose medium, with 10\% FBS and $1 \%$ of Antibiotics (GIBCO, Invitrogen). Cells were purified and expanded by serial passaging until their characterization and use in the xenotransplant experiments, as performed for UC-MSC.

Expression of typical MSC cell-surface markers was evaluated by flow cytometry. Adherent cells were trypsinized, washed, and incubated with the following antihuman primary antibodies: CD29-PECy5, CD34-PerCP, CD31-phycoerythrin (PE), CD45-fluorescein isothiocyanate (FITC), CD90-R-PE, CD73-PE, CD13-PE, CD44-PE, CD117-PE, human leukocyte antigen (HLA)-ABC-FITC, HLA-DR-R-PE (Becton, Dickinson and Company, Franklin
Lakes, NJ). A total of 10,000 labeled cells were analyzed using a Guava EasyCyte flow cytometer running Guava ExpressPlus software (Guava Technologies Hayward, CA). (Supplemental Figure 1).

\section{Experimental Model of PD}

Male adult Wistar rats (200-250 g) were housed under standard controlled conditions (7:00 AM/7:00 P.M. light/ dark cycle; $20-22^{\circ} \mathrm{C} ; 45-55 \%$ humidity) with food and water ad libitum. All efforts were made to minimize animal suffering following the proposal of International Ethical Guideline for Biomedical Research (CIOMS/OMS, 1985). The study was approved by the Ethics Committee for Animal Research of the Federal University of São Paulo, Brazil (CEP 2071/07).

Experimental animals ( $n=10$ per group) were subjected to a toxin-induced model of PD. Damage to the nigrostriatal dopaminergic system was induced with the neurotoxin MPTP-HCl (1-Methyl-4-phenyl-1,2,3,6-tetrahydropyridine hydrochloride - Sigma-Aldrich, St. Louis) dissolved in saline $(0,9 \% \mathrm{NaCl})$ at a final concentration of $2 \mathrm{mg} / \mathrm{mL}$. MPTP was infused via intranasal with a peristaltic pump set at a flaw rate of $12.5 \mu \mathrm{L} / \mathrm{min}$, as previously described [23]. A PE-50 catheter was inserted $8 \mathrm{~mm}$ deep into the nasal cavity. The inhalation lasted four minutes per nostril $(0.1 \mathrm{mg} / 50 \mu \mathrm{L})$, with $1 \mathrm{~min}$ interval between each inhalation. Control animals received the same amount of saline only.

\section{Cell Transplantation Protocol}

One week after MPTP or saline treatment, animals were subjected to intracranial infusion of either vehicle (sham), human fibroblasts (cellular control), human UC-MSC, or a mixture of $50 \%$ UC-MSC and $50 \%$ fibroblasts $(n=10$, per experimental group). Animals were anesthetized with sodium thiopental $(75 \mathrm{mg} / \mathrm{kg}$, i.p.) and a concentrated suspension of cells $\left(10^{5}\right.$ in $5 \mu \mathrm{L}$ of DMEM-LG without FBS) was injected with a high precision $10 \mu \mathrm{L}$ microsyringe (Hamilton Co.) in the right striatum (Bregma $+0.70 \mathrm{~mm} \mathrm{AP},+3.0 \mathrm{~mm} \mathrm{ML},-4.5 \mathrm{~mm} \mathrm{DV}$ ) or in the right ventricle (Bregma $-0.80 \mathrm{~mm}$ AP, $-1.5 \mathrm{~mm} \mathrm{ML},-3.5 \mathrm{~mm}$ DV) through a stereotaxic brain surgery. Sham animals received $5 \mu \mathrm{L}$ of DMEM-LG without FBS only. Motor deficits and brain histology were evaluated 4 weeks after initial exposure to MPTP or saline (i.e. 3 weeks after cell transplantation).

Behavioral Assessment

Different behavioral tests were applied to address the effects of MPTP administration on motor activity and 
emotionality of experimental animals. Hypokinesia was determined based on exploratory activities and locomotion measured by the open field test [24]. Animals were positioned in the center of a circular arena $(100 \mathrm{~cm}$ diameter, $40 \mathrm{~cm}$ high) divided in 18 squares, and the number of squares crossed by the rats was registered for 5 min. Catalepsy and bradykinesia were determined based on the animal's ability to correct its posture and time of immobility in the tail suspension test, respectively [25]. Animals were placed in a head-down position and the capacity and latency to orient in the head-up position were video recorded. Anxiety was evaluated by the elevated-plus maze test. The maze was comprised by two open arms $(29 \mathrm{~cm} \times 5 \mathrm{~cm})$, two closed arms $(29 \mathrm{~cm} \times 5 \mathrm{~cm} \times 15 \mathrm{~cm})$, and a central region $(5 \mathrm{~cm} \times 5 \mathrm{~cm})$. Animals were positioned in the center of the maze and behavior was observed for $5 \mathrm{~min}$. Anxiety levels were determined by the percentage of time spent in the open arms relative to the total time spent in both open and closed arms [26].

Data were analyzed by Two-way ANOVA with Bonferroni as post-hoc test. All conclusions were based on at least $5 \%$ level of significance $(P \leq 0.05)$.

\section{Immunofluorescence}

Human cells were detected in rat brains by immunofluorescence using antibodies specific to human DNA. Animals were deeply anesthetized with sodium pentobarbital $(75 \mathrm{mg} /$ $\mathrm{kg}$ i.p.) and perfused transcardially with $\mathrm{PBS} \mathrm{pH} 7.4$ for $30 \mathrm{~min}$ (12 $\mathrm{mL} / \mathrm{min}$ rate), followed by $4 \%$ paraformaldehyde in PBS for $40 \mathrm{~min}$. Brains were transferred to a $4 \%$ paraformaldehyde solution for $24 \mathrm{~h}$, cryoprotected in a $30 \%$ sucrose solution for 3 days, and sectioned at $70 \mu \mathrm{m}$ on a cryostat. Tissue sections were blocked with 10\% FBS, 5\% bovine serum albumin (BSA), and $0.1 \%$ Triton $\mathrm{X}-100$ in PBS for $1 \mathrm{~h}$ at room temperature and incubated at $4{ }^{\circ} \mathrm{C}$ overnight with primary antibody (mouse anti-human nuclei CAT\# MAB1281, Chemicon International, California, USA) at a 1:20 dilution, followed by incubation with secondary anti-mouse $\mathrm{IgG}$ antibody at a 1:100 dilution for $2 \mathrm{~h}$ at room temperature. Tissues were counterstained with $5 \mu \mathrm{g} / \mathrm{mL} \mathrm{4}$, 6Diamidino-2-phenylindol (DAPI) and microscope slides mounted in Vectashield medium (Vector Laboratories). Immunofluorescence analysis was performed in a Zeiss Imager Z1 Apotome microscope with epi-fluorescence, or using an argon ion laser scan microscope LSM 410 (Zeiss Jena, Germany). Images were captured and digitalized with the Axiovision 4.8 software.

\section{Quantification of Dopaminergic Neurons}

Brain sections were cut at a $70 \mu \mathrm{m}$ thickness using a vibratome (Leica VT 1000s) and dopaminergic neurons were detected with antibody against tyrosine hydroxylase (TH) (ABCAM, cat\# ab113). The sampled sections were chosen according to the systematic random sampling scheme, with a section sampling fraction (ssf) of $1 / 5$. After quenching endogenous peroxidases with $3 \% \mathrm{H}_{2} \mathrm{O}_{2}$ in PBS for $10 \mathrm{~min}$, sections were incubated for $2 \mathrm{~h}$ with $10 \% \mathrm{BSA} /$ $0,3 \%$ Triton $\mathrm{X}-100$, and then incubated with primary antibody at a 1: 2000 dilution overnight at $4{ }^{\circ} \mathrm{C}$, followed by incubation with biotinylated anti-mouse $\operatorname{IgG}$ (Vector) at 1:200 dilution for $2 \mathrm{~h}$ at room temperature. Immunostaining was performed with 90-min incubation in avidin-biotin peroxidase complex (ABC Vector $\mathrm{Lab}$ ) and detection in $0.075 \%$ DAB (diaminobenzidine, Sigma-Aldrich) with $30 \% \mathrm{H}_{2} \mathrm{O}_{2}$.

$\mathrm{TH}+$ neurons were estimated by stereology using the optical-fractionator design [27]. This method includes the use of an optical dissector at regularly predetermined $\mathrm{x}, \mathrm{y}$ axis within the SN of Wistar rat. The SN was delineated using $1 \times$ magnifications according to the delimitations given by Paxinos (1995) [28]. Step " $x$ " was predetermined to $200 \mu \mathrm{m}$ and step "y" to $200 \mu \mathrm{m}$. Microscopic (Carl Zeiss, Imager M1, Germany) images obtained from SN using $63 \times$ objective captured and an unbiased counting frame was then superimposed using the Stereoinvestigator 8.21 software (MicroBrightField, Colchester, VT, USA). The counting frame area of the dissector $(70 \mu \mathrm{m} \times 70 \mu \mathrm{m}=$ $4900 \mu \mathrm{m} 2$ ) was focused through $70 \mu \mathrm{m}$ thickness and the number of neurons counted with unbiased counting rules. The height of the dissector was $10 \mu \mathrm{m}$ for this study. At each step in the sampled cell, the distance between the top and bottom surfaces were determined [29], indicating that shrinkage is homogeneous within each studied section. All sections after processing and mounting were on average $17 \mu \mathrm{m}( \pm 1.85$ s.d.) thick. The fraction of the sampled section thickness is referred to as the thickness sampling fraction (tsf) $=\mathrm{h}$ (the height of the disector)/t (the mean thickness of the section).

$\mathrm{TH}+$ neurons were directly counted in the established fraction of the SN. Coefficient of error (CE) for cell number estimates was calculated from the formula:

$C E(N)=\sqrt{C E^{2}+C E^{2}(t)^{2}}$

adopting the one-stage systematic sampling procedure (CE Scheaffer-Mendenhall-Ott) that has been applied and validated elsewhere [30].

We also estimated the total volume ( $\mathrm{Vol})$ in the $\mathrm{SN}$ with the Cavalieri method:

$V_{o l} l_{r e f}=t \times a(x, y-$ step $) \times \frac{1}{s s f} \times \Sigma P$

where $\mathrm{t}$ is the mean section thickness, $\sum \mathrm{P}$ is the sum of upper right corner points of the counting frames that lie 
within the region of interest, and a is the area associated with the $\mathrm{x}, \mathrm{y}$-step lengths.

\section{Results}

Engraftment of Human Cells into the Brains of Immunocompetent Rats

Prior to the in vivo trial, two intracranial infusion routes were evaluated with regards to human cell engraftment. Identical amounts of human UC-MSC were infused either in the right lateral ventricle or locally in the right striatum. The injection in the ventricular system was attempted to facilitate cell distribution to the main components of the basal ganglia, including striatum and SN. However, 1 week post- intracerebroventricular (i.c.v.) administration of UCMSC, only a few isolated cells could be detected in rat brains, mainly in the ipsilateral striatum relative to the injection site. Similar distribution pattern was observed in rats previously exposed to MPTP. One week after i.c.v. administration, a limited amount UC-MSC could be observed in the vicinity of the right ventricle. When human fibroblasts were injected via i.c.v., engrafted cells were rarely detected in brains of control and MPTP-treated rats (Fig. 1a-c).

Alternatively, when directly injected into the right striatum, a greater amount of grafted UC-MSC could be detected in rat brains even 3 weeks after cell administration. UC- MSC could be detected not only in the vicinity of the injection site, where they were present at a high density, but also in other brain regions such as the medial septal nucleus, peripheral zone of the striatum, and in the $\mathrm{SN}$ (Fig. 1d-i). Noteworthy, at the striatum, UC-MSC were detected in both ipsilateral and contralateral sites. Interestingly, the existence of neurodegeneration due to previous exposure to MPTP did not change this distribution pattern. Similar results were obtained in animals that inhaled either saline or MPTP 1 week prior to cell injection. As observed with the i.c.v. route, when injected in the striatum, biodistribution of human fibroblasts was similar to that of UC-MSC, even though fewer engrafted cells could be detected (Fig. 2).

\section{Intrastriatal Infusion of UC-MSC Preserves \\ Dopaminergic Neurons}

Since human cell grafts were more efficiently formed through intrastriatal cell infusion, this administration route was chosen to further examine potential therapeutic effects of UC-MSC in rats subjected to the experimental model of PD induced by MPTP. One week post-MPTP or -saline (control) inhalation, animals were infused with vehicle
Fig. 1 Engraftment of human UC-MSC and fibroblasts in rat brains. Cells were injected either in the right cerebral ventricle $(\mathbf{a}-\mathbf{c})$ or directly into the right striatum (d-o) of control and MPTP-injured rats. Human cells could be detected by immunofluorescence 1 week post-i. c.v. injection and 3 weeks post-intrastriatal injection. The white arrows indicate examples of cells that reacted positively with DAPI (blue fluorescence) and antibody specific to human DNA (red fluorescence). UC-MSC in MPTP-injured rats $(\mathbf{a}, \mathbf{b}, \mathbf{d}-\mathbf{f})$, UC-MSC in control rats $(\mathbf{g}-\mathbf{i})$, fibroblasts in MPTP-injured rats $(\mathbf{c}, \mathbf{j}-\mathbf{l})$, fibroblasts in control rats $(\mathbf{m}-\mathbf{0})$. Bar scale $50 \mu \mathrm{m}$

(sham), human UC-MSC, human UC-MSC plus human fibroblasts $(50 \%$ each), or human fibroblasts (as a cellular control). Immunohistochemical analysis of dopaminergic $(\mathrm{TH}+)$ neurons revealed a marked bilateral neurodegeneration in the SN, 4 weeks after MPTP exposure (Fig. 3). Tyrosine hydroxylase is a rate-limiting enzyme in dopamine synthesis that localizes in the cytosol and synaptic termini. Qualitative analysis of control-sham rat brains showed a cluster of $\mathrm{TH}+$ neurons in the $\mathrm{SN}$ with many $\mathrm{TH}+$ termini in the background (Fig. 3a-b). In addition to neuronal loss, the remaining $\mathrm{TH}+$ neurons in the $\mathrm{SN}$ of sham rats exposed to MPTP displayed a different cytoarchitecture characterized by citoplasmatic retraction and scarce $\mathrm{TH}+$ synaptic termini (Fig. 3c-d).

However, animals exposed to MPTP and later treated with UC-MSC displayed preserved dopaminergic neurons in the SN. Both cytoarchitecture and immunohistochemical staining pattern were similar to those found in the SN of control animals. Interestingly, such preservation of dopaminergic neurons was also observed in the contralateral SN relative to the cell infusion site. Conversely, similar neuropreservation was neither found in fibroblast-treated nor in UC-MSC plus fibroblasttreated animals that had been previously exposed to MPTP (MPTP-fibroblasts, and MPTP-UC-MSC/fibroblasts, respectively).

Further quantitative analysis by stereology confirmed neurodegeneration induced by MPTP and neuropreservation in rats subsequently treated with UC-MSC. As shown in Fig. 4, rats exposed to MPTP (MPTP-sham) contained nearly half $\mathrm{TH}+$ neurons in the $\mathrm{SN}$ after 4 weeks, compared with control animals (control-sham). Under the same time frame, rats that received an infusion of UC-MSC 1 week after MPTP exposure had significantly higher amounts of $\mathrm{TH}+$ neurons in the SN than MPTP-sham, MPTP-UCMSC/fibroblasts, and MPTP-fibroblast rats. Such amount was equivalent to that found in control-sham animals and is in agreement with the amount detected in normal rats [31]. Histological analysis did not detect intracranial tumors in animals infused either with UC-MSC or fibroblasts (Supplemental Figure 2). 

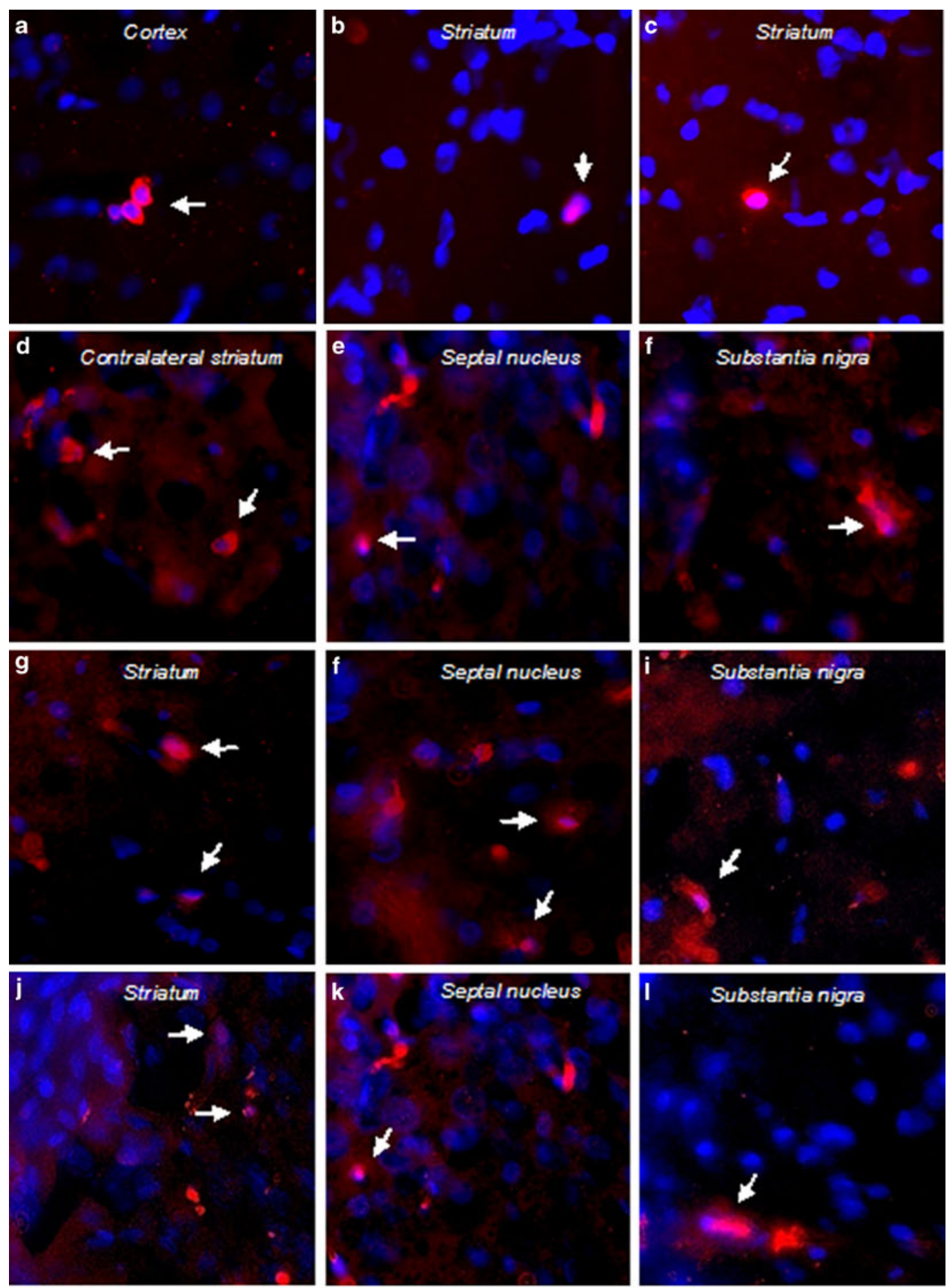

m

Striatum

4
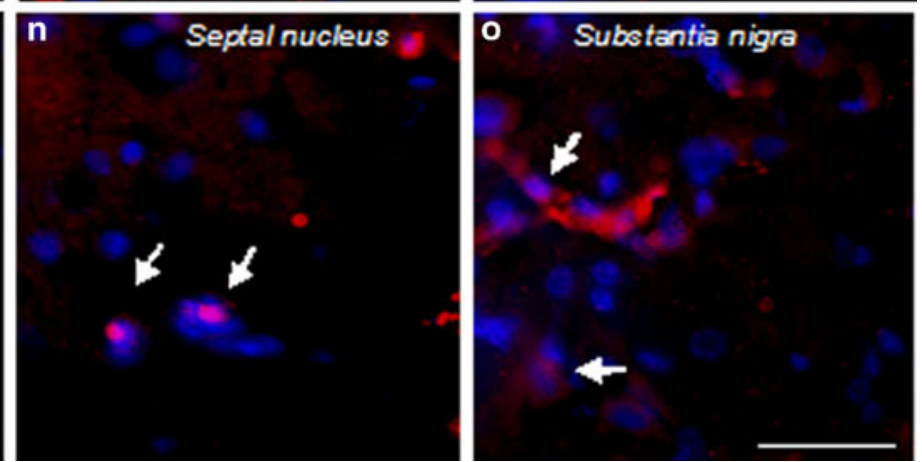
Intrastriatal Infusion of Human Fibroblasts Induces Neurodegeneration in the SN

The stereological analysis of SN also revealed a significant reduction in the amount of $\mathrm{TH}+$ neurons in animals that received an infusion of human fibroblasts. As indicated in Supplemental Table 1, fibroblast-treated animals had SN of similar volume but with significantly reduced quantity of $\mathrm{TH}+$ neurons than animals of all other experimental groups. Such reduction was observed both in control and MPTP-exposed animals, attaining similar amounts of $\mathrm{TH}+$ neurons 3 weeks from the fibroblast injection $\left(5.1 \times 10^{3}\right.$ and $4.7 \times 10^{3}$ cells, respectively). Such amounts correspond to about $34-37 \%$ the amount of $\mathrm{TH}+$ neurons present in the $\mathrm{SN}$ of control-sham animals. Surprisingly, the reduction in $\mathrm{TH}+$ neurons induced by intrastriatal human fibroblast injection was significantly higher than the reduction caused by MPTP neurotoxicity alone, suggesting a potent neurodegenerative effect. Noteworthy, significant degeneration of $\mathrm{TH}+$ neurons after injection of UC-MSC alone or UC-MSC plus fibroblasts were also found in control animals.

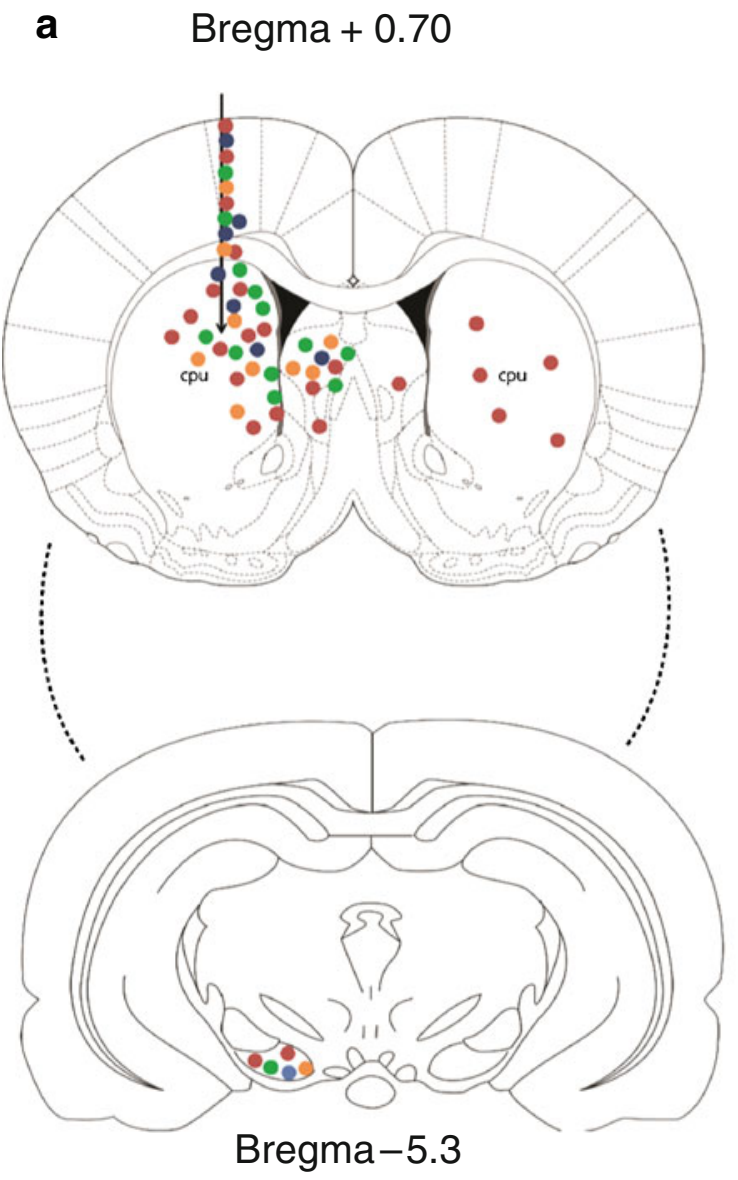

Fig. 2 Scheme illustrating intracerebral distribution of engrafted human cells. a Intrastriatal administration, b i.c.v. administration. The arrows indicate the site of cell injection. Colored circles indicate major
Prevention of Motor Deficits in MPTP-Injured Rats Due to UC-MSC Treatment is Reverted by Fibroblast Contamination

MPTP neurotoxicity in rats caused motor deficits that were evident 4 weeks after the MPTP inhalation. Compared with control-sham animals, MPTP-sham animals presented symptoms of hypokinesia, indicated by significant lower locomotor activity measured by the open field test. Symptoms of catalepsy and bradykinesia were also detected 4 weeks after MPTP inhalation, indicated respectively by a significant lower capability of posture recovery and increased immobility time (Fig. 5a-c). Fibroblast transplantation in control animals also caused significant hypokinesia and catalepsy. Hypokinesia, but not catalepsy and bradykinesia, was also verified in control animals transplanted with UC-MSC.

Conversely, MPTP-exposed animals that were treated with UC-MSC displayed indexes of locomotion, reflex, and time of immobility that were comparable to those observed in control-sham animals. No significant signs of hypokinesia, catalepsia, and bradykinesia were observed in the

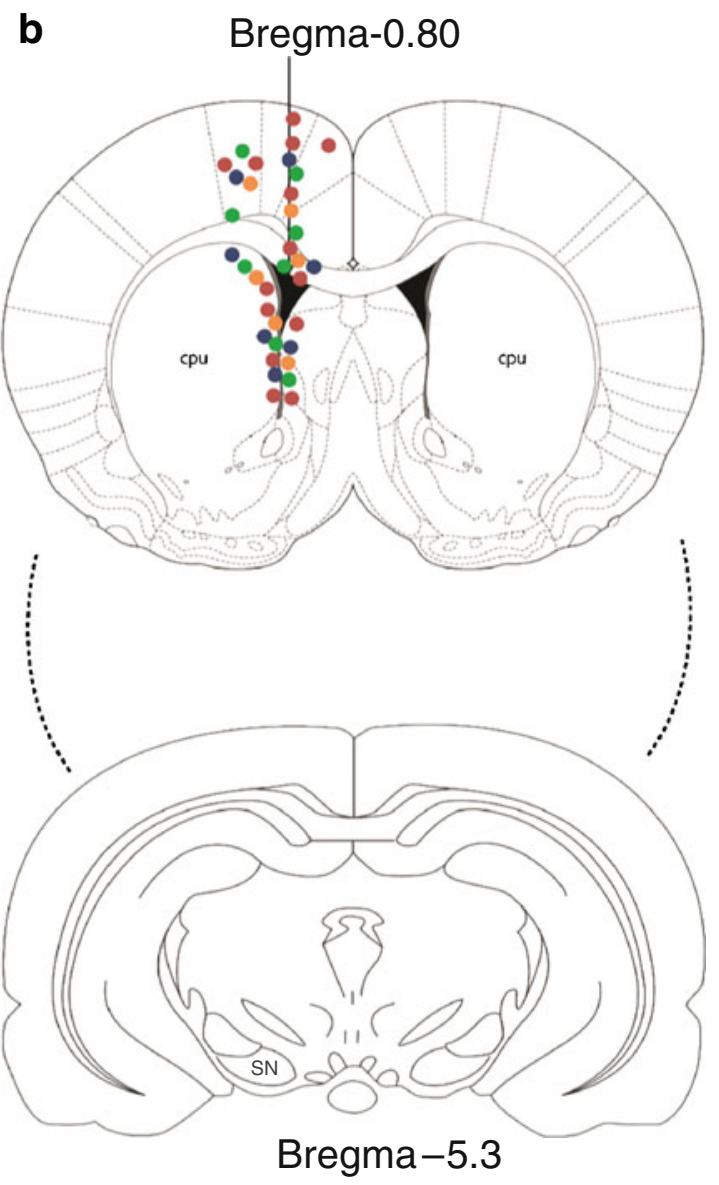

locations of engrafted human cells within rat brains. Green: Control UC-MSC, red: MPTP - UC-MSC, orange: Control - Fibroblast, blue: MPTP - Fibroblast. $\mathrm{Cpu}=$ caudatus putamen; $\mathrm{SN}=$ substantia nigra 


\section{CONTROL}
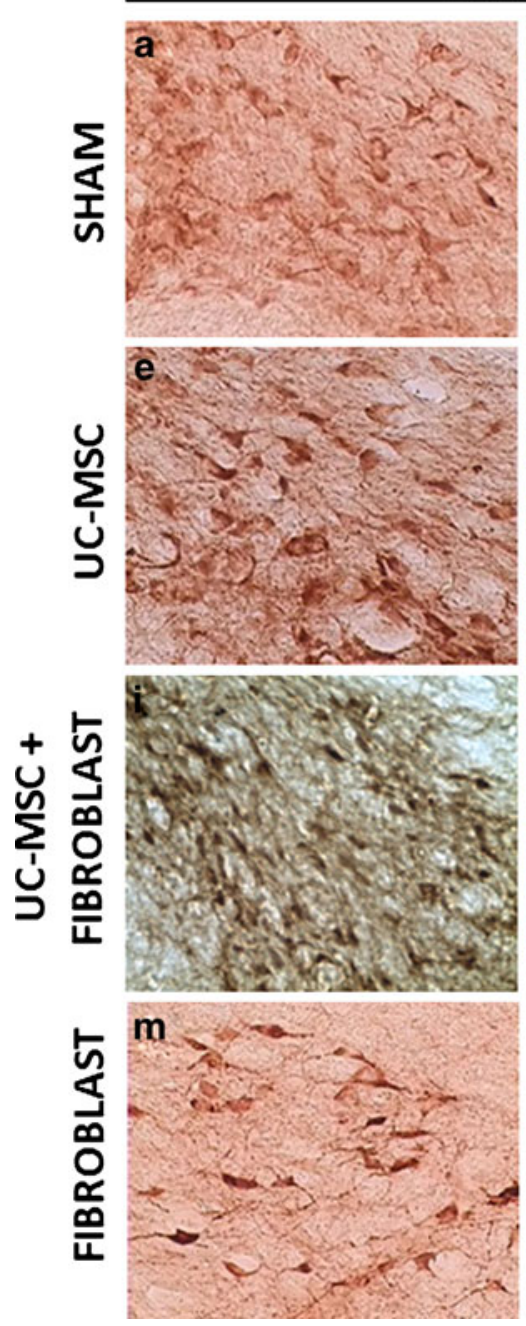
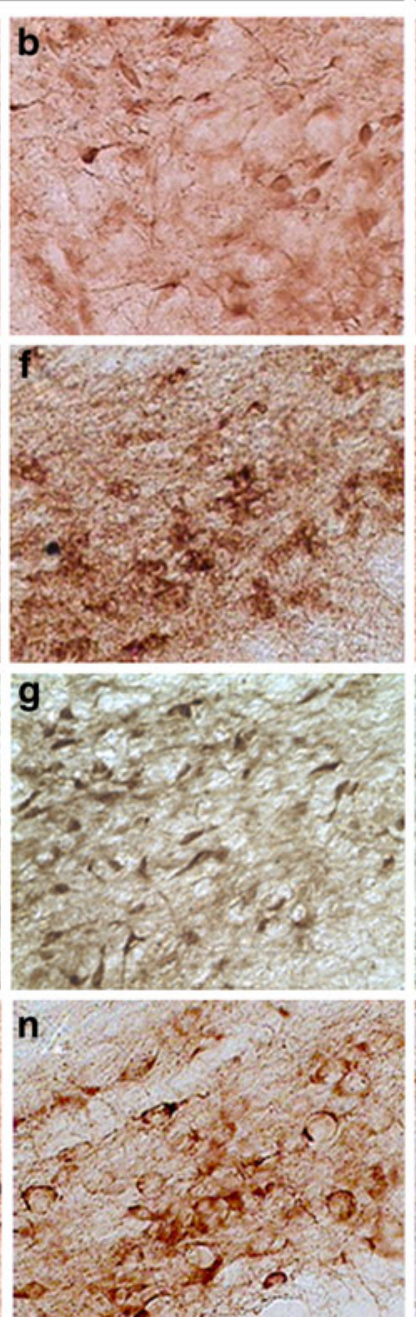

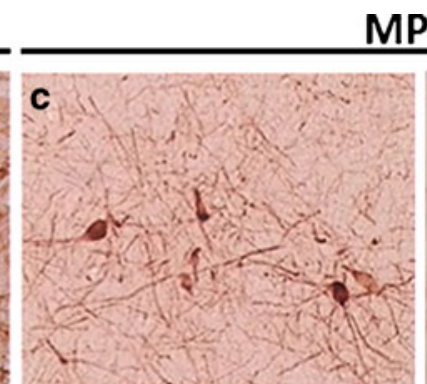

MPTP
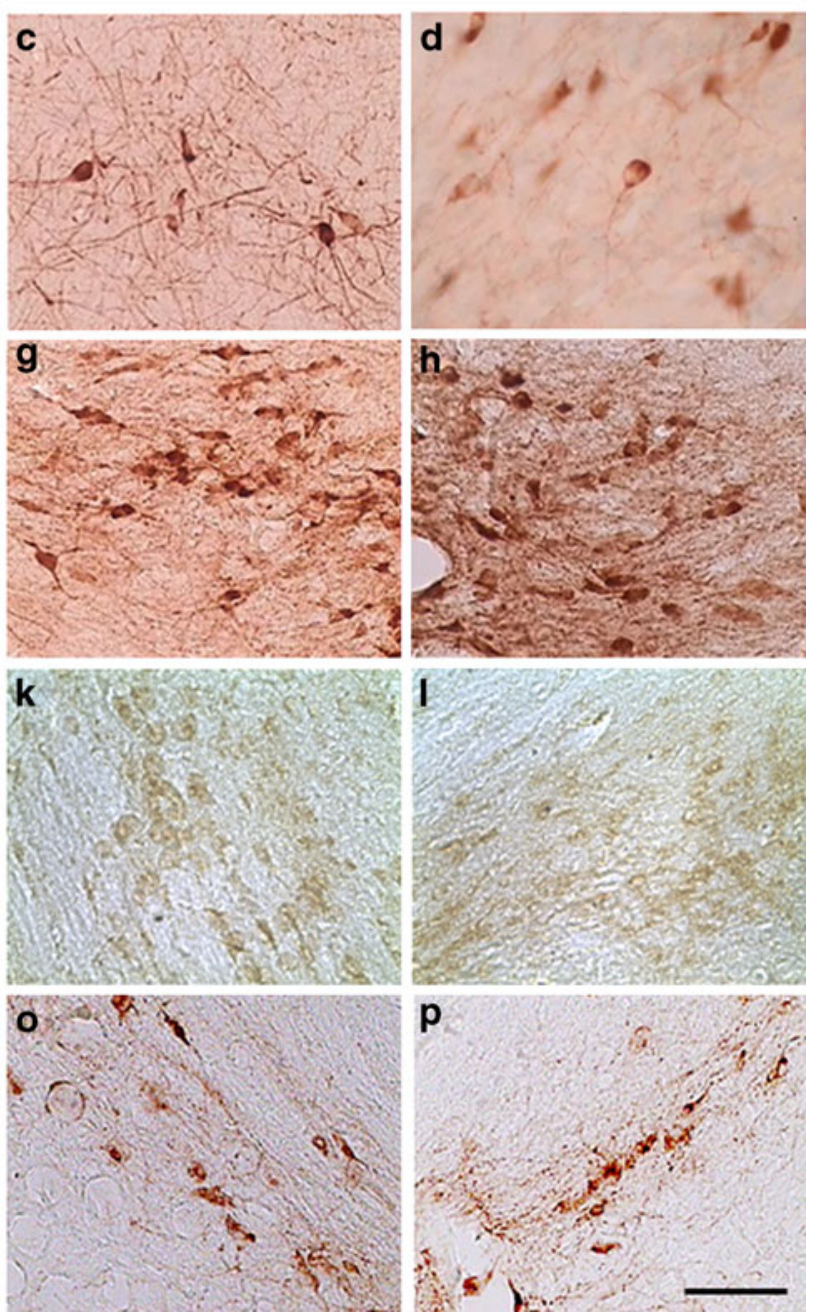

Fig. 3 Population of dopaminergic neurons in the SN of rats indicated by immunohistochemistry with anti-tyrosine hydroxylase. Rats inhaled either saline (control) or MPTP and 1 week later were subjected to an intrastriatal injection of vehicle (sham), human UCMSC and/or human fibroblasts. Immunohistochemical analyzes were performed 4 weeks post injury with MPTP (i.e. 3 weeks post intrastriatal injections). TH+ neurons at both ipsilateral $(\mathbf{a}, \mathbf{c}, \mathbf{e}, \mathbf{g}, \mathbf{i}$, $\mathbf{k}, \mathbf{m}, \mathbf{o})$ and contralateral $(\mathbf{b}, \mathbf{d}, \mathbf{f}, \mathbf{h}, \mathbf{i}, \mathbf{l}, \mathbf{n}, \mathbf{p})$ sites relative to intrastriatal injection are shown. Control-Sham (a, b), MPTP-Sham (c, d), Control-UC-MSC (e, f), MPTP - UC-MSC (g, h), Control-UCMSC/fibroblast (i, j), MPTP-UC-MSC/fibroblast (k, l), Controlfibroblast $(\mathbf{m}, \mathbf{n})$, MPTP-fibroblast $(\mathbf{o}, \mathbf{p})$. Bar scale $50 \mu \mathrm{m}$
Fig. 4 Amount of $\mathrm{TH}+$ neurons in the SN of control and MPTPinjured rats. One week post MPTP inhalation, rats were subjected to an intrastriatal injection of vehicle (sham), human UC-MSC and/or human fibroblasts. Control animals inhaled saline. Data represent mean values quantified 4 weeks post injury with MPTP. Statistical significance: $* P \leq 0.05$,

** $P \leq 0.01, * * * P \leq 0.005$

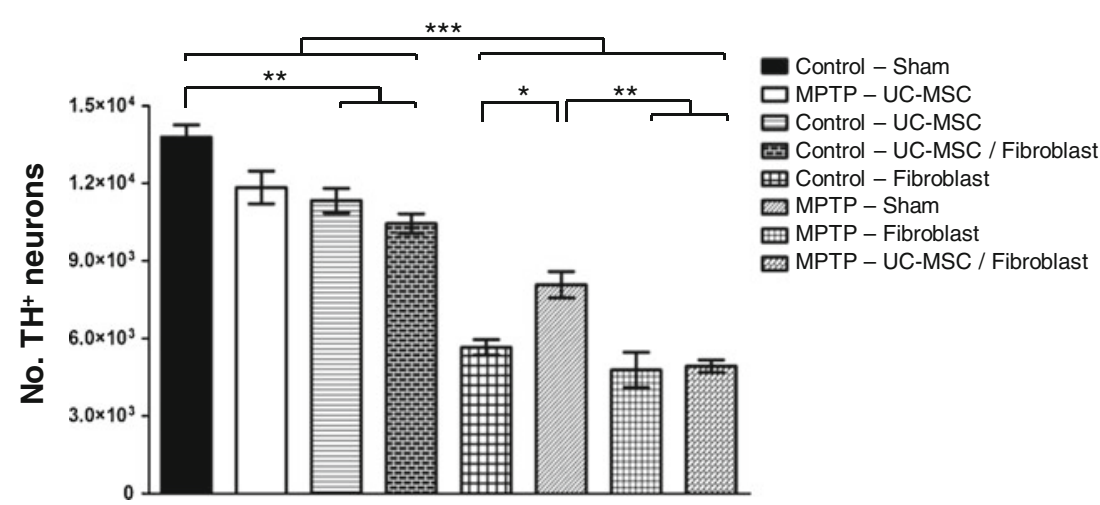



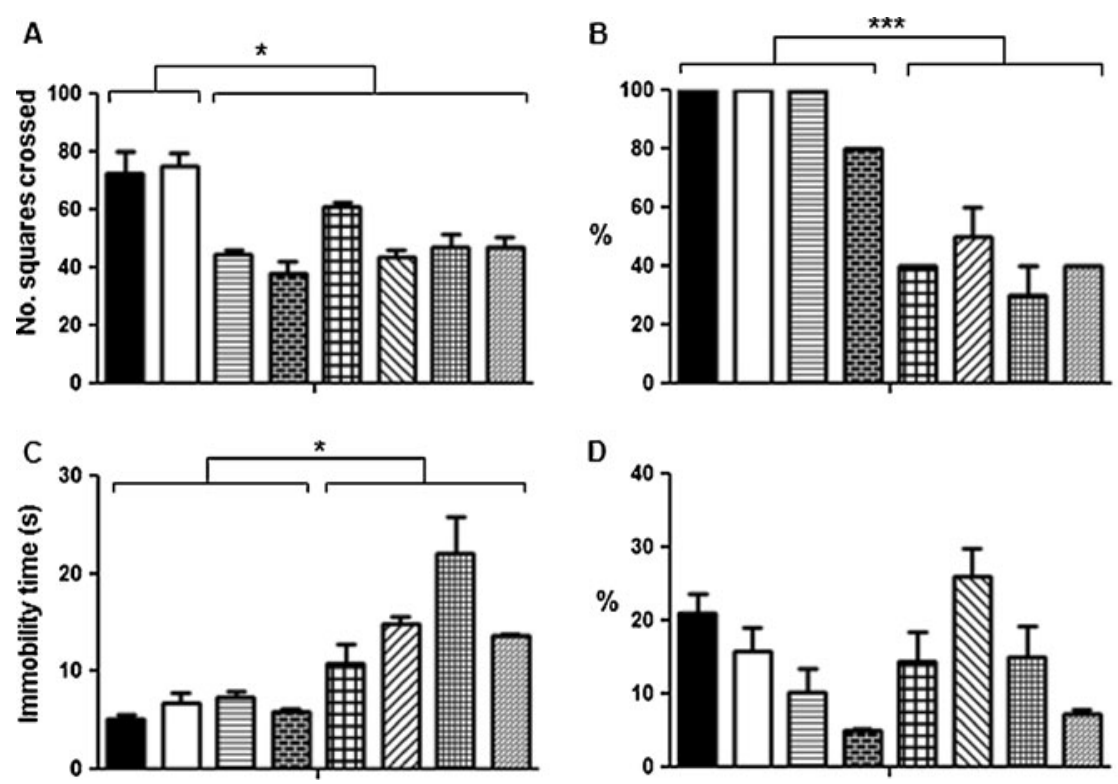

D

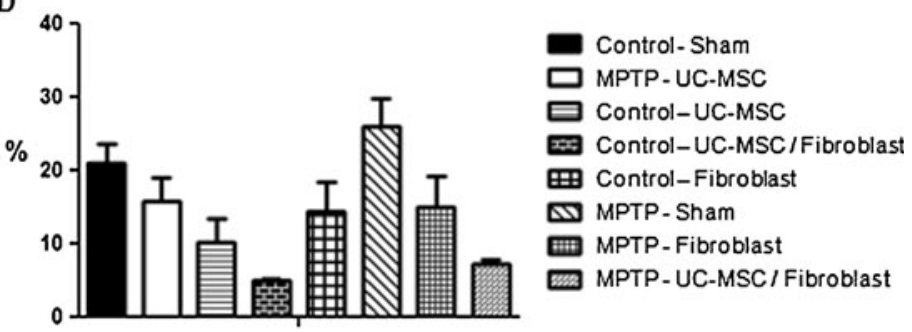

Fig. 5 Motor activity of rats subjected to the MPTP model of PD. Rats inhaled either saline (control) or MPTP and 1 week later were subjected to an intrastriatal injection of vehicle (sham), human UCMSC and/or human fibroblasts. Behavioral tests were performed 4 weeks post injury with MPTP. a Hypokinesia based on locomotor

UC-MSC-treated animals. More importantly, such therapeutic effects of UC-MSC could not be observed when they were mixed with fibroblasts. The behavioral tests detected significant differences in the motor activity of MPTP-UCMSC animals compared with MPTP-sham, MPTP-UCMSC/fibroblasts and MPTP-fibroblasts animals. No significant differences were noted in the anxiety levels of animals due to MPTP exposure or human cell transplantation (Fig. 5d).

\section{Discussion}

Mesenchymal stem cells from human cord are emerging as suitable candidates for use in cell therapy protocols due to their multipotency and immunomodulatory properties. From the practical viewpoint, UC-MSC can be isolated at high yields and in vitro expanded by simple procedures that are feasible to adapt in a hospital routine. The existing infrastructure for cryopreservation of umbilical cord blood may also facilitate banking of the entire umbilical cord unit for future clinical applications [21]. However, here we show that the lack of proper quality control and poor purity of MSC preparations may induce adverse as opposed to benefitial effects and affect overall clinical outcome. In this work, the therapeutic properties of UC-MSC were further evaluated in an experimental model of PD, revealing novel findings with clinical implications. activity determined by the open field test. b Catalepsy determined by the percentage of animals capable of recovering position on the tail suspension test, $\mathbf{c}$ Bradykinesia based on time of immobility in the tail suspension test, d Anxiety evaluated by the elevated-plus maze test. Statistical significance: $* P \leq 0.05, * * * P \leq 0.005$

Our first observation was that intracranial infusion of $\mathrm{UC}-\mathrm{MSC}$ in the lateral ventricle did not facilitate cell engraftment in components of the basal ganglia. Since asymmetrical lateral ventricular enlargement is associated with motor asymmetry and progression in PD [32], injection of cells in the ventricular system would be a convenient approach allowing cell contact with different brain regions affected in PD including striatum, pallidum, substantia nigra, and subthalamic nucleus, as well as the cerebral cortex. However, UC-MSC were rarely seen in rat brains 1 week after i.c.v. administration. Only a few isolated cells were detected, mostly in the striatum.

In fact, cell engraftment and biodistribution were improved by direct injection of cells in the right striatum. Through this administration route, cells could even be detected in the contralateral striatum and other brain structures distant from the injection site, including the SN, indicative of cell migration. Migration of MSC within the brain has also been reported in hemiparkinsonian rats injured with 6-hydroxy-dopamine [8]. Neurotrophic factor-secreting MSC isolated from rat [10] and human [11] bone marrow that were transplanted into rat striatum have been shown to migrate from the striatum and corpus callosum toward sites of injury. While our results confirmed the ability of MSC to migrate within the brain and reach the $\mathrm{SN}$, no indications of preferential cell homing to brain injured tissue were observed. The biodistribution of UCMSC after either i.c.v. or intrastriatal injections were similar in control rats and in rats with neurodegeneration caused by 
previous exposure to MPTP. Since UC-MSC were inoculated 1 week after MPTP inhalation, active cell homing through chemoattraction might possibly involve factors secreted in earlier stages of neurodegeneration. Previous reports of active MSC homing also indicate requirement of supplementary neurotrophic brain factors $[10,11]$. In any event, these results suggest that intracranial transplantation of MSC close to injured sites may be more advantageous in clinical trials than systemic administration.

The second observation was that, even though present in small quantities in the rat brain, UC-MSC were capable of protecting the dopaminergic neurons in the $\mathrm{SN}$ against the neurotoxic effects of MPTP. This observation is supported by the similar amounts of $\mathrm{TH}+$ neurons found in the $\mathrm{SN}$ of control rats (i.e. not exposed to MPTP) and rats exposed to MPTP but subsequently treated with UC-MSC. More importantly, this neuroprotection effect occurred bilaterally and was specifically caused by the UC-MSC grafts since the same phenomenon was not observed in MPTP-exposed rats that were later infused with either vehicle or human fibroblasts, where a significant degeneration of $\mathrm{TH}+$ neurons occurred in SN. A recent study with modified human bone marrow MSC transplanted in the striatum of hemiparkinsonian rats injured with 6-hydroxy-dopamine [11] reported that most MSC were destroyed by the rat immune system 1 week post-injection, although a small amount of cells could still be detected 50 days postinjection. A similar situation was found in the present study, suggesting that even small amounts of remaining human MSC in the host's brain may exert a potent neuroprotector effect. The fact that neuropreservation in SN occurred bilaterally further reinforces this point.

Furthermore, the neuroprotector effect caused by UCMSC transplantation also prevented motor deficits resulting from the MPTP neurotoxicity. In fact, besides inducing a bilateral neurodegeneration in the $\mathrm{SN}$, another advantage of the MPTP model of PD is that it allows the use of different behavioral tests to address motor function. In the present study, rats exposed to MPTP and subsequently treated with UC-MSC did not show motor dysfunctions typically observed in PD patients such as hypokinesia, catalepsy, and bradykinesia, as opposed to MPTP-exposed rats that were infused either with vehicle or fibroblasts. Such specific functional effects of UC-MSC were detected as early as 3 weeks after cell transplantation, emphasizing a potent therapeutic effect. Intracranial transplantation of UCMSC did not generate tumors and did not cause effects related to anxiety or depression in rats.

The third observation was provided by the additional cellular control of xenografted cells included in the present study which revealed that transplantation of human cells without stem cell properties may be deleterious. Intrastriatal transplantation of human fibroblasts in rats induced a significant degeneration of $\mathrm{TH}+$ neurons in the $\mathrm{SN}$ that resulted in motor deficits. While engraftment of human UCMSC in the brain of immunocompetent rats was expected due to the immunossupressor activity of MSC, human fibroblast grafts had not been anticipated. This could be partially explained by the fact that the experiments involved a single intracranial cell injection and that the brain is an immunoprivileged organ protected by the hematoencefalic barrier. Nonetheless, given that fewer fibroblasts were detected in rat brains than UC-MSC, the significant reduction in $\mathrm{TH}+$ neurons in the $\mathrm{SN}$ of fibroblast-treated rats is indicative of a potent neurotoxic effect.

More importantly, presence of fibroblasts as a cell contaminant in the UC-MSC preparation abolished the above mentioned therapeutic effects of stem cell transplantation and further enhanced degeneration of dopaminergic neurons. Since fibroblasts do not share the same immunomodulatory properties of MSC, the observed neurodegeneration could be partly explained by xenograft rejection. In a clinical scenario, although such rejection would not occur in autotransplantation, abolishment of therapeutic effects by fibroblasts could considerably affect clinical outcome. However, when using human umbilical cord as a source of MSC, allotransplants are likely to be more frequent than autotransplants. In this scenario, the presence of contaminant allogeneic fibroblasts may induce inflammation and a local immune response.

Indeed, in a preliminary clinical trial with PD patients subjected to allotransplantation of mesencephalic tissue, Olanow et al. [4] reported inflammation and activated microglia around brain grafts, indicating immune reaction. Such immune rejection of brain allografts were reported to be involved in the degeneration of dopaminergic neurons and occurrence of diskinesias observed in some PD patients [7]. Since inflammation and immune reaction within the brain are known to contribute to neurodegeneration in PD, as well as in other prevalent neurodegenerative diseases [33], contamination of MSC preparations with fibroblasts should be carefully controlled in allogeneic transplantation.

Recent advances in stem cell research include the identification of a variety of stem cell types coming from alternative biological sources or technological procedures, which pose the challenge of understanding the advantages and disadvantages of each particular cell type to pursue prospective therapeutic applications. The therapeutic effects observed in rats treated with UC-MSC are comparable to those reported with transplantation of neurons that had been differentiated from embryonic stem cells and iPS cells in experimental models of PD [15, 16, 34]. However, instead of direct reconstitution of dopaminergic neurons alone, the mechanisms of UC-MSC-mediated neuroprotection may also involve paracrine effects towards an improved microenvironment in the central nervous system, favoring restoration in detriment of degeneration [35, 36]. The 
discrepancy in the amount of engrafted UC-MSC in the SN and the respective extensive preservation of $\mathrm{TH}+$ neurons quantified by stereology, in addition to the bilateral neuroprotector effect observed, all support this hypothesis.

Intriguingly, partial recovery of $\mathrm{TH}+$ neurons has been reported in rats lesioned with 6-hydroxy-dopamine, albeit only 12 weeks post MSC transplant, a time when engrafted cells could no longer be detect in rat brains [9], suggesting mobilization of endogenous neuroprogenitors. However, the intrinsic mechanism involved is currently unclear and should be further addressed.

\section{Conclusion}

This pre-clinical study of cell therapy in PD reveals potent and specific effects of intrastriatal transplantation of human UC-MSC regarding bilateral preservation of dopaminergic neurons in the SN and prevention of motor deficits. Such therapeutic effects, however, were reversed and an enhanced neurodegeneration was observed when the UC-MSC preparation was also comprised by fibroblasts, a mesenchymal cell type without stem cell properties.

The neurotoxic effect observed due to transplantation of human fibroblasts suggests that purity of cell preparations must be carefully considered in clinical trials, in particular those involving allotransplantation, to avoid possible severe adverse effects. Cell population heterogeneity may also explain different results observed in previous pre-clinical and clinical trials. Another distinctive aspect of the present study is that cell transplantation was performed 1 week after MPTP exposure, when dopamine deficiency and neurodegeneration are still progressing and motor activity is not fully affected [23]. Since this experimental situation is more associated with the initial phase of $\mathrm{PD}$, our findings provide a rationale for clinical trials of UC-MSC transplantation with the aim of delaying disease progression and ensuing intensification of motor deficits, through neuroprotective homeostatic adjustments in the brain.

\begin{abstract}
Acknowledgments This work was supported by grants and fellowships from CAPES, CNPq, INCT Células-tronco em Doenças Genéticas Humanas and FAPESP-CEPID. The authors also thank Iara R. Silva and Ariadne S. Gonçalves for assistance in experiments and behavioral tests.
\end{abstract}

Conflicts of interest The authors declare no potential conflicts of interest.

Open Access This article is distributed under the terms of the Creative Commons Attribution Noncommercial License which permits any noncommercial use, distribution, and reproduction in any medium, provided the original author(s) and source are credited.

\section{References}

1. Lindvall, O., Brundin, P., Widner, H., et al. (1990). Grafts of fetal dopamine neurons survive and improve motor function in Parkinson's disease. Science, 247, 574-577.

2. Freed, C. R., Breeze, R. E., Rosenberg, N. L., et al. (1992). Survival of implanted fetal dopamine cells and neurologic improvement 12 to 46 months after transplantation for Parkinson's disease. New England Journal of Medecine, 327, 1549-1555.

3. Freed, C. R., Geene, P. E., Breeze, R. E., et al. (2001). Transplantation of embryonic dopamine neurons for severe Parkinson's disease. New England Journal of Medecine, 344, 710-719.

4. Olanow, C. W., Goetz, C. G., Kordower, J. H., et al. (2003). A double-blind controlled trial of bilateral fetal nigral transplantation in Parkinson's disease. Annals of Neurology, 54, 403-414.

5. Isacson, O. (2009). Cell therapy ahead for Parkinson's disease. Science, 326, 358-359.

6. Preynat-Seauve, O., Burkhard, P. R., Villard, J., et al. (2009). Pluripotent stem cells as new drugs? The example of Parkinson's disease. Internacional Journal Pharmaceutics, 381, 113-121.

7. Lindvall, O., \& Kokaia, Z. (2009). Prospects of stem cell therapy for replacing dopamine neurons in Parkinson's disease. Trends in Pharmacological Sciences, 30, 260-267.

8. Fu, Y., Cheng, Y., Anya, L. M., et al. (2006). Conversion of human umbilical cord mesenquimal stem cells in Wharton's Jelly to dopaminergic neurons in vitro: potencial terapeutic application for Parkinsonism. Stem Cells, 24, 115-124.

9. Weiss, M. L., Medicetty, S., Bledsoe, A. R., et al. (2006). Human umbilical cord matrix stem cells: preliminary characterization and effect of transplantation in a rodent model of Parkinson's disease. Stem Cells, 24, 781-792.

10. Sadan, O., Shemesh, N., Barzilay, R., et al. (2008). Migration of neurotrophic factors-secreting mesenchymal stem cells towards a quinolinic acid lesion as viewed by MRI. Stem Cells, 26, 2542 2551.

11. Sadan, O., Bahaat-Stromza, M., Barhun, Y., et al. (2009). Protective effects of neurotrophic factors-secreting cells in a 6OHDA rat model of Parkinson's Disease. Stem Cells and Development, 18, 1-12.

12. Roy, N. S., Cleren, C., Singh, S. K., et al. (2006). Functional engraftment of human ES cell-derived dopaminergic neurons enriched by coculture with telomerase-immortalized midbrain astrocytes. Nature Medicine, 12, 1259-1268.

13. Ravindran, G., \& Rao, H. S. (2006). Enriched NCAM-positive cells form functional dopaminergic neurons in the rat model of Parkinson's disease. Stem Cells and Development, 15, 575-582.

14. Bjugstad, K. B., Teng, Y. D., Redmond, D. E. J., et al. (2008). Human neural stem cells migrate along the nigroestriatal pathaway in a primate model of Parkinson's Disease. Experimental Neurology, 211, 362-369.

15. Cho, M. S., Lee, Y. E., Kim, J. Y., et al. (2008). Highly efficient and large-scale generation of functional dopamine neurons from human embryonic stem cells. Proceedings of the National Academy of Sciences, 105, 3392-3397.

16. Wernig, M., Zhao, J. P., Pruszak, J., et al. (2008). Neurons derived from reprogrammed fibroblasts functionally integrate into the fetal brain and improve symptoms of rats with Parkinson's Disease. Proceedings of the National Academy of Sciences, 105, 58565861.

17. Park, I. H., Arora, N., Huo, H., et al. (2008). Disease-specific induced pluripotent stem cells. Cell, 134, 877-886.

18. Wei, P., Liu, J., Zhou, H., et al. (2007). Effects of engrafted neural stem cells derived from GFP transgenic mice in Parkinson's diseases rats. Neuroscience Letters, 419, 49-54. 
19. Li, J. Y., Englund, E., Holton, J. L., et al. (2008). Lewy bodies in grafted neurons in subjects with Parkinson's disease suggest hostto-graft disease propagation. Nature Medicine, 14, 501-503.

20. Secco, M., Zucconi, E., Vieira, N. M., et al. (2008). Multipotent stem cells from umbilical cord: cord is richer than blood! Stem Cells, 26, 146-150.

21. Secco, M., Moreira, Y. B., Zucconi, E., et al. (2009). Gene expression profile of mesenchymal stem cells from paired umbilical cord units: cord is different from blood. Stem Cell Reviews, 5, 387-401.

22. Morandi, F., Raffaghello, L., Bianchi, G., et al. (2008). Immunogenicity of human mesenchymal stem cells in HLA-class Irestricted T-cell responses against viral or tumor-associated antigens. Stem Cells, 26, 1275-1287.

23. Prediger, R. D. S., Batista, L. C., Medeiros, R., et al. (2006). The risk is in the air: Intranasal administration of MPTP to rats reproducing clinical features of Parkinson's disease. Experimental Neurology, 202, 391-403.

24. Fredriksson, A., \& Archer, T. (1994). MPTP-induced behavioural and biochemical deficits: a parametric analysis. Journal of Neural Transmission Parkinson Disease and Dementia Section, 7, 123-132.

25. Cryan, J. F., Mombereau, C., \& Vassout, A. (2005). The tail suspension test as a model for assessing antidepressant activity: review of pharmacological and genetic studies in mice. Neurocience Biobehavioral Reviews, 29, 571-625.

26. Frussa-Filho, R., Otoboni, J. R., Uema, F. T., et al. (1991). Evaluation of memory and anxiety in rats observed in the eleveted plus-maze: effects of age and isolation. Brazilian Journal of Medicine and Biological Research, 24, 725-728.

27. West, M. J., Slomianka, L., \& Gundersen, H. J. (1991). Unbiased stereological estimation of the total number of neurons in the subdivisions of the rat hippocampus using the optical fractionator. The Anatomical Record, 231, 482-497.

28. Paxinos, G., \& Watson, C. (1995). The rat brain: In stereotaxic coordinates (2nd ed.). San Diego: Academic.

29. Korkmaz, A., \& Tümkaya, L. (1997). Estimation of the section thickness and optical disector height with a simple calibration method. Journal of Microscopy, 187, 104-109.

30. Glaser, E. M., \& Wilson, P. D. (1998). The coefficient of error of optical fractionator population size estimates: a computer simulation comparing three estimators. Journal of Microscopy, 192, 163-171.

31. Hardman, C. D., Henderson, J. M., Finkelstein, D. I., et al. (2002). Comparison of the basal ganglia in rats, marmosets, macaques, baboons, and humans: volume and neuronal number for the output, internal relay, and striatal modulating nuclei. The Journal of Comparative Neurology, 445, 238-255.

32. Lewis, M. M., Smith, A. B., Styner, M., et al. (2009). Asymmetrical lateral ventricular enlargement in Parkinson's disease. European Journal of Neurology, 16, 475-481.

33. Amor, S., Puentes, F., Baker, D., \& van der Valk, P. (2010). Inflammation in neurodegenerative diseases. Immunology, 129, 154-169.

34. Bjorklund, L. M., Sanchez-Pernaute, R., Chung, S., et al. (2002). Embryonic stem cells develop into functional dopaminergic neurons after transplantation in a Parkinson rat model. Proceedings of the National Academy of Sciences, 99, 2344-2349.

35. Crigler, L., Robey, R. C., Asawachaicharm, A., et al. (2006). Human mesenchymal stem cell subpopulations express a variety of neuro-regulatory molecules and promote neuronal cell survival and neuritogenesis. Experimental Neurology, 198, 54-64.

36. Lee, P. J., Baek, R. C., Clark, D., et al. (2007). Stem cells act through multiple mechanisms to benefit mice with neurodegenerative metabolic disease. Nature Medicine, 13, 439-447. 